\title{
Criticality and Scaling Relations in a Sheared Granular Material
}

\author{
Takahiro Hatand* \\ Earthquake Research Institute, University of Tokyo, 113-0032, Japan \\ Michio Otsuk $\oplus$ and Shin-ichi Sasa $\ddagger$ \\ Department of Pure and Applied Sciences, University of Tokyo, Komaba, Tokyo 153-8902, Japan
}

(Dated: May 6, 2018)

\begin{abstract}
We investigate a rheological property of a dense granular material under shear. By a numerical experiment of the system with constant volume, we find a critical volume fraction at which the shear stress and the pressure behave as power-law functions of the shear strain rate. We also present a simple scaling argument that determines the power-law exponents. Using these results, we interpret a power-law behavior observed in the system under constant pressure.
\end{abstract}

PACS numbers: 47.57.Gc,47.50.-d,45.50.-j

\section{INTRODUCTION}

Soft glassy systems such as foams, colloidal suspensions, emulsions, polymers, glasses [1, 2, 3], and granular materials [4, 5, 6] have a strongly non-linear response to an external perturbation. In such systems, the relation between the stress $\sigma$ and the strain rate $\gamma$ characterizes the system behavior. Although it is known that the relations are diverse and specific to individual systems, a universal law for a certain class of systems may exist.

In particular, in sheared granular materials under constant pressure $p$, one of the authors (Hatano) has found a relation 7 ]

$$
\frac{\sigma}{p} \simeq I^{\phi}
$$

with

$$
I=\sqrt{\frac{m}{p a}} \gamma
$$

by a numerical experiment using the discrete element method. Here, $a$ is the maximum diameter of the particles (their diameters are uniformly distributed in the range $[0.7 a, a])$ and $m$ is the mass of the particles [8]. As demonstrated in Fig. 11 the exponent $\phi$ is not inconsistent with $1 / 5$ in the range $10^{-3} \leq I \leq 10^{-1}$. Surprisingly, the power-law behavior given in Eq. (1) is observed in the cases that $p / Y \simeq 10^{-3}$ and $10^{-5}$, where $Y$ represents the Young modulus of the particle. For example, one can experimentally obtain the power-law behavior under the constant pressure $p=10^{0}-10^{-2} \mathrm{MPa}$ by using polystyrene with $Y=3 \mathrm{GPa}$. Since $I=10^{-3}$ corresponds to the shear rate $10^{0}-10^{1} / \mathrm{sec}$ in this example, the shear condition leading to Eq. (11) is experimentally possible.

\footnotetext{
*Electronic address: hatano@eri.u-tokyo.ac.jp

${ }^{\dagger}$ Electronic address: otsuki@jiro.c.u-tokyo.ac.jp

${ }^{\ddagger}$ Electronic address: sasa@jiro.c.u-tokyo.ac.jp
}

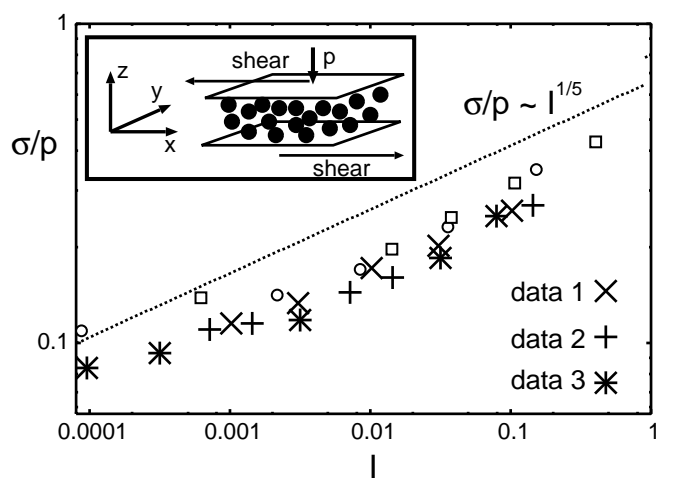

FIG. 1: $\sigma / p$ as a function of $I$. This result was obtained for a model similar to that explained in the text. The main differences are as follows: (i) the top boundary in the $z$ direction is modified so as to maintain a constant pressure and (ii) the shear is applied directly from the moving layer at the top and the bottom. (See the inset.) The parameter values are as follows: $L_{x} / a=L_{y} / a=25, N=10800$, and $\eta / \sqrt{\mathrm{km}}=1.0 . \mathrm{pa} / \mathrm{k}=1.92 \times 10^{-3}($ data 1$), 3.75 \times 10^{-5}($ data 2 ), and $p a / k=4.35 \times 10^{-3}$ with $L_{x} / a=L_{y} / a=15$ (data $3)$. Furthermore, the square and circle symbols represent the constant pressure data obtained from Figs. 2] and [3] where $p a / k=\Pi=1.25 \times 10^{-3}$ (square symbol) and $5.4 \times 10^{-5}$ (circle symbol).

Stimulated by this result, in the present paper, we consider the power-law behavior of stress-strain rate relations in sheared granular materials by investigating a model granular system with the Lees-Edwards boundary conditions. In this idealized system, we demonstrate that there is a critical volume fraction at which the shear stress and the pressure (normal stress) behave as powerlaw functions of the shear strain rate in the limit $\gamma \rightarrow 0$. From these power-law behaviors, we derive the scaling relation $\sigma / p \simeq I^{1 / 5}$ in the limit $I \rightarrow 0$ at the critical volume fraction. Note that this critical condition does not correspond to a constant pressure. We then present a simple interpretation of Eq. (1) for the system under constant pressure. 


\section{MODEL AND NUMERICAL RESULTS}

Here, we describe our computational model. The system consists of $N$ spheres of mass $m$ in a threedimensional rectangle box whose lengths are $L_{x}, L_{y}$, and $L_{z}$ along the $x, y$, and $z$ directions, respectively. In order to realize an average velocity gradient $\gamma$ in the $z$ direction and average velocity in the $x$ direction, we impose the Lees-Edwards boundary conditions [9]. The particle diameters are $0.7 a, 0.8 a, 0.9 a$ and $a$ each of which is assigned to $N / 4$ particles. When the distance between two particles is less than the sum of their radii, $r_{1}$ and $r_{2}$, an interaction force acts on each of them. This force comprises an elastic repulsion force $k\left(\delta r-\left(r_{1}+r_{2}\right)\right)$ and the viscous dissipation force $\eta \delta v$, where $\delta r$ and $\delta v$ represent the relative distance and velocity difference of the interacting particles, respectively. For simplicity, we do not consider the tangential force between the interacting particles. We study the specific case where $L_{x} / a=L_{y} / a=L_{z} / a, N=1728$ and $\eta / \sqrt{k m}=1.0$. The control parameters in this system are the volume fraction $\nu \equiv \sum_{i=1}^{N} \pi a_{i}^{3} /\left(6 L_{x} L_{y} L_{z}\right)$ with the $i$ th particle diameter $a_{i}$, and the dimensionless shear rate $\Gamma \equiv \gamma \sqrt{m / k}$. We then calculate the dimensionless shear stress $\Sigma=\sigma a / k$ and the dimensionless pressure (in the $z$ direction) $\Pi=p a / k$. See Ref. [9] as the calculation method for $\Sigma$ and $\Pi$. Note that $k / a$ provides an approximate value of the Young modulus of particles.

We express the dependence of $\Sigma$ and $\Pi$ on $(\Gamma, \nu)$ as $\Sigma=f_{\sigma}(\Gamma, \nu)$ and $\Pi=f_{p}(\Gamma, \nu)$, respectively. Figures 2 and 3 display these functions with respect to $\Gamma$ for several values of $\nu$ 10]. These graphs clearly show that there exists a critical volume fraction $\nu_{\mathrm{c}}$ at which the power law behaviors are observed as follows:

$$
\begin{aligned}
& f_{\sigma}\left(\Gamma, \nu_{\mathrm{c}}\right) \simeq \Gamma^{\alpha}, \\
& f_{p}\left(\Gamma, \nu_{\mathrm{c}}\right) \simeq \Gamma^{\beta},
\end{aligned}
$$

in the limit $\Gamma \rightarrow 0$ [1]. The values of the exponents will be discussed later. Here, it is worthwhile noting that similar graphs were obtained in Ref. [10] with the argument on the effect of finite elastic modulus. Indeed, these graphs in this reference suggest the existence of the critical state, although the power-law behavior was not mentioned explicitly. Upon numerical verification, we found that the critical volume fraction corresponds to the jamming transition point defined as the volume fraction beyond which a finite yield stress appears [12]. In this paper, we do not argue the nature of the jamming transition, but focus on the power-law behaviors given in Eqs. (3) and (4). Note that a similar critical state was obtained for a sheared glassy system [1].

\section{THEORETICAL ARGUMENT}

The main idea in our theoretical argument is to consider dimensional analysis with kinematic temperature $T$

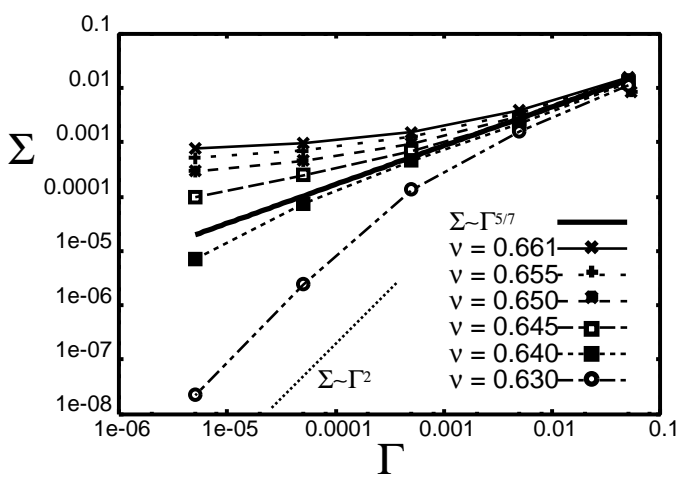

FIG. 2: $\Sigma$ as a function of $\Gamma$ for several values of $\nu$. The thick solid line represents $\Sigma \propto \Gamma^{5 / 7}$ that is estimated from our theoretical argument. Note that the Bagnold scaling [13] is observed for the case in which $\nu=0.630$ and $\Gamma \leq 10^{-4}$.

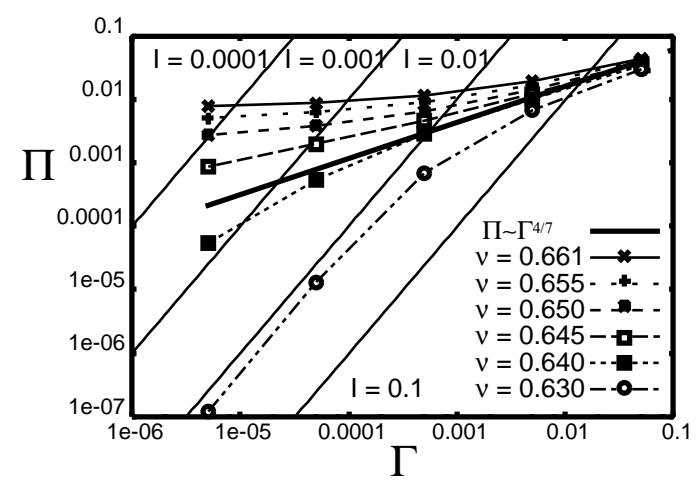

FIG. 3: $\Pi$ as a function of $\Gamma$ for several values of $\nu$. The thick solid line represents $\Pi \propto \Gamma^{4 / 7}$ that is estimated from our theoretical argument. Furthermore, the lines $I=$ const. are drawn to help us to understand the functional form of $\sigma / p$ over $I$.

defined as

$$
T=\frac{m}{3 N}\left(\sum_{i=1}^{N}\left\langle\left|\boldsymbol{v}_{i}\right|^{2}\right\rangle-\left|\left\langle\boldsymbol{v}_{i}\right\rangle\right|^{2}\right),
$$

where $\boldsymbol{v}_{i}$ denotes the velocity of the $i$-th particle. Although $T$ is not a parameter of the system but is determined by $\Gamma$ and $\nu$, it is considered that physical processes in granular systems are described in terms of the kinematic temperature [14]. In particular, the time scale of energy dissipation is assumed to be determined as $(\sqrt{T / m} / a)^{-1}$. One can verify the validity of this assumption by investigating the energy balance equation in the steady state [14]:

$$
\frac{N}{L_{x} L_{y} L_{Z}} \frac{T^{3 / 2}}{a \sqrt{m}} \simeq \sigma \gamma,
$$


which is rewritten as

$$
\nu\left(\frac{T}{k a^{2}}\right)^{3 / 2} \simeq \Sigma \Gamma
$$

Figure 4 indicates that Eq. (17) is plausible as the first theoretical attempt, although a slight deviation is observed. Based on this result, hereafter, we assume that the time scale of the energy dissipation is given by $(\sqrt{T / m} / a)^{-1}$.

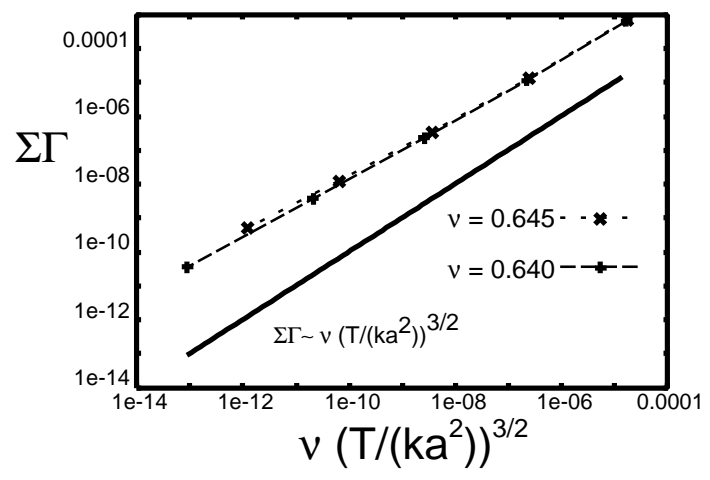

FIG. 4: $\quad \nu\left(T /\left(k a^{2}\right)\right)^{3 / 2}$ versus $\Sigma \Gamma$ for two cases in which $\nu$ is close to the critical volume fraction $\nu_{\mathrm{c}}$. The solid line corresponds to Eq. (7). Since we find the best fitting to be $\nu\left(T /\left(k a^{2}\right)\right)^{3 / 2} \sim(\Sigma \Gamma)^{0.9}$, there is a slight deviation from Eq. (7).

Now, we consider a set of dimensionless constants using the energy dissipation ratio. Let us recall that we have three independent dimensionless parameters $(\nu, \Gamma, \eta / \sqrt{k m})$. We want to use dimensionless parameters each of which represents the ratio of a time scale of a physical process to that of energy dissipation. It is then reasonable that the following two time scales are important: the inverse of the shear rate $\gamma^{-1}$ and the relaxation time $\eta / k$ of the elastic displacement during the interaction between two particles. Thus, instead of $\Gamma$ and $\eta / \sqrt{\mathrm{km}}$, we introduce

$$
\begin{aligned}
\xi_{1} & =a \sqrt{\frac{m}{T}} \gamma, \\
\xi_{2} & =\frac{1}{k a} \sqrt{\frac{T}{m}} \eta .
\end{aligned}
$$

Then, we wish to determine the functional forms of $\Sigma\left(\xi_{1}, \xi_{2}\right)$ and $\Pi\left(\xi_{1}, \xi_{2}\right)$ in the limit $\Gamma \rightarrow 0$ at the critical volume fraction. In order to restrict the possible forms of the functions, we further assume that $\sigma$ does not depend on $k$. Noting that $\xi_{2} \rightarrow 0$ for $\Gamma \rightarrow 0$, the function becomes

$$
\Sigma \simeq A\left(\xi_{1}\right) \xi_{2}
$$

where $A\left(\xi_{1}\right)$ represents the direct contribution of $\Gamma$ that is not expressed through the dependence of $T$ on $\Gamma$.
Here, we consider $A\left(\xi_{1}\right)$ on the basis of the theory determining the behavior of shear stress near the critical state for a dense colloidal suspension 15]. According to this theory, the critical behavior is described by an order parameter equation that is similar to the GinzburgLandau equation for magnetization under a magnetic field. Assuming that this description is valid for the present problem, we write

$$
c_{0}\left(\nu-\nu_{\mathrm{c}}\right) A\left(\xi_{1}\right)-c_{1} A\left(\xi_{1}\right)^{3}=\xi_{1}
$$

with numerical constants $c_{0}$ and $c_{1}$. Note that, in cases of magnetic materials, $A\left(\xi_{1}\right)$ and $\xi_{1}$ correspond to magnetization and a magnetic field, respectively. Because the first term vanishes at the critical volume fraction, we obtain

$$
A\left(\xi_{1}\right) \simeq \xi_{1}^{1 / 3} .
$$

Following these assumptions, we can calculate the exponents $\alpha$. Concretely, Eq. (10) with Eq. (12) becomes

$$
\Sigma \simeq \xi_{1}^{1 / 3} \xi_{2} .
$$

Combining Eq. (13) with Eq. (7), we derive

$$
T \simeq a^{2} m^{1 / 7} \eta^{6 / 7} \gamma^{8 / 7} .
$$

The substitution of this into Eq. (13) with Eqs. (8) and (9) yields

$$
\Sigma \simeq\left(\frac{\eta}{\sqrt{k m}}\right)^{9 / 7} \Gamma^{5 / 7} .
$$

Thus, we obtain $\alpha=5 / 7$ in Eq. (3), which is consistent with the numerical experiment as shown in Fig. 2 In a similar manner, we obtain

$$
\Pi \simeq\left(\frac{\eta}{\sqrt{k m}}\right)^{10 / 7} \Gamma^{4 / 7}
$$

under the assumption that $\Pi\left(\xi_{1}, \xi_{2}\right) \simeq \xi_{2}$. This assumption implies that $\Pi$ does not depend on $\xi_{1}$ because the normal stress is not directly influenced by the shear rate. As shown in Fig. 3] $\beta=4 / 7$ in Eq. (4) is consistent with the numerical experiment.

\section{INTERPRETATION OF EQ. (1)}

We next study the power-law behavior observed in the system under constant pressure on the basis of the results obtained above. First, from Eqs. (3) and (4), which are valid at the critical volume fraction $\nu_{\mathrm{c}}$, we derive Eq. (10) with

$$
\phi=\frac{2(\alpha-\beta)}{2-\beta} .
$$


Using the values $\alpha=5 / 7$ and $\beta=4 / 7$, we obtain $\phi=$ $1 / 5$. This value of $\phi$ is consistent with the numerical experiment. However, it should be noted that the scaling relation is obtained at the critical volume fraction, not for systems under constant pressure.

In order to discuss quantitatively the behavior of the system under constant pressure, we denote the volume fraction and the shear stress measured on this system as $\nu=g_{\nu}(\Gamma, \Pi)$ and $\Sigma=g_{\sigma}(\Gamma, \Pi)$. We then wish to determine these functions from Figs. 2] and 3 First, the point $(\Gamma, \Pi)$ in Fig. [3 determines the volume fraction uniquely. We assume here that this volume fraction is realized in the system under constant pressure $\Pi$ with shear rate $\Gamma$ and that the shear stress in the system is determined by using the volume fraction in Fig. 2 Note that this assumption was confirmed directly by a numerical experiment for a constant pressure system whose size is close to that of the system with the Lees-Edwards boundary conditions. Based on this assumption, we determine the volume fraction as a function of $\Gamma$ at a constant pressure. In addition, using this, we can obtain the dependence of the shear stress on $\Gamma$ at a constant pressure. Hence, we obtain $\sigma / p$ as a function of $I$ under constant $\Pi$. For reference, we plot the lines $I=$ const. in Fig 3 As an example in Fig. [3 let us consider the case that $\Pi=10^{-3}$ in which the volume fraction is larger than the critical one for a sufficiently small $\Gamma$. This case corresponds to the regime $I \leq 10^{-3}$ and $\nu \geq 0.650$. Then, from Fig. 2 we find that the shear stress remains almost constant. Next, in the interval $10^{-3} \leq I \leq 10^{-1}$, the states with $\Pi=10^{-3}$ are close to the critical line. Thus, in this regime, it is expected that $\sigma / p$ behaves as that in the critical line, and the scaling behavior given in Eq. (10) is observed approximately.

Generalizing the above discussion, we expect the typical dependence of $\sigma / p$ on $I$ as follows:

$$
\begin{aligned}
\sigma / p & \approx \text { const. for } I \ll I_{0}, \\
& \approx I^{1 / 5} \text { for } I_{0}<I<I_{1},
\end{aligned}
$$

when spatially homogeneous shear flow is realized. Note that $I_{0}$ and $I_{1}$ are dependent on the pressure. For example, for states with extreme pressures, such relations would not be observed. Since we wish to know the extent to which this approximate power-law relation holds, in Fig. 1 we include the constant pressure data $(I, \sigma / p)$ obtained from Figs. 2 and 3 As expected from the above consideration, the power-law behavior is observed for the case in which $\Pi=1.25 \times 10^{-3}$. Furthermore, the system obeys the power-law regime even for the case $\Pi=5.4 \times 10^{-5}$ in which the line is located below the critical states in Fig. 3. We do not understand the reason why the power law regime is so wide.

\section{CONCLUDING REMARK}

We have presented numerically and theoretically the scaling relations given in Eqs. (3) and (4) for the system with the Lees-Edwards boundary conditions. From these new scaling relations, we also have an interpretation of the result observed in the system under constant pressure. The result is summarized in Eqs. (18) and (19).

As far as we know, few experimental results exist in this regard. We expect that the power-law behaviors given in Eqs. (31) and (41) are observed in systems with constant volume, e.g., by operating a rotating Coutteflow system [16]. With regard to Eq. (18) that is valid in the very low shear rate regime, we conjecture that a thermal activation process, which might lead to the logarithmic dependence of $\sigma / p$ on $I$, occurs in this regime. Note that such behavior is ubiquitous in shear flow and sliding friction [17]. Furthermore, the result reported in Ref. 18 might be related to Eq. (19). We hope that more intensive experimental studies will be performed in this regard.

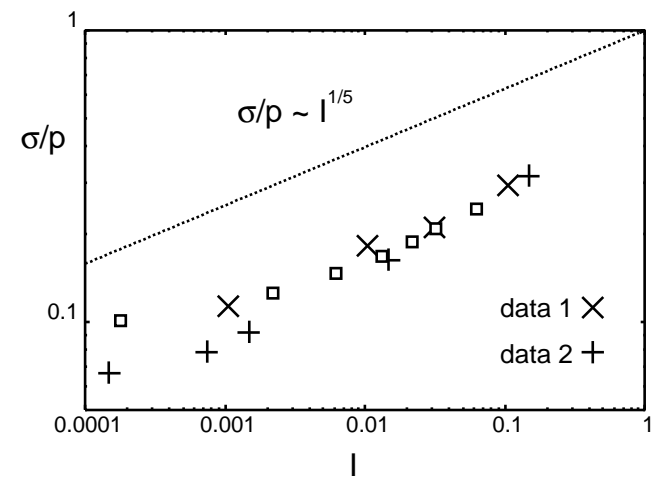

FIG. 5: $\quad \sigma / p$ as a function of $I$ for the model described in the caption of Fig. 11 with a Herzian type interaction $k^{\prime}(1-$ $\left.\delta r /\left(r_{1}+r_{2}\right)\right)^{3 / 2}$. The parameter values are as follows: $L_{x} / a=$ $L_{y} / a=25, N=10800$, and $\eta \sqrt{a} / \sqrt{k^{\prime} m}=1.0 . p a^{2} / k^{\prime}=$ $3.75 \times 10^{-5}$ (data 1$), 1.92 \times 10^{-3}$ (data 2). Furthermore, the square symbols represent the constant pressure data obtained from Figs. 6] and 7] where the interaction force is $k^{\prime \prime}\left(\left(r_{1}+\right.\right.$ $\left.\left.r_{2}\right)-\delta r /\right)^{3 / 2}$ and $p \sqrt{a} / k^{\prime \prime}=6.625 \times 10^{-4}$.

Related to experimental studies, one may be interested in the dependence of our result on the choice of the model we investigate. For example, one may choose the Herzian type as an alternative for the interaction force. Indeed, such a model dependence has been discussed in the case of zero-temperature and zero applied stress [4]. In our problem, it is highly expected that there is a critical state at which rheological properties exhibit power-laws. However, it is not evident that the exponents remain the same values for the model with a Herzian type interaction.

In order to consider the model dependence explicitly, we demonstrate the result of numerical experiments in Figs. 5 6 and 7 It is seen that the exponent of the system under constant pressure does not deviate so much from $1 / 5$, while the exponents $\alpha$ and $\beta$ seem to change slightly. We do not have a theoretical understanding for these values yet, because the time scale related to the particle collision is not directly determined from model 


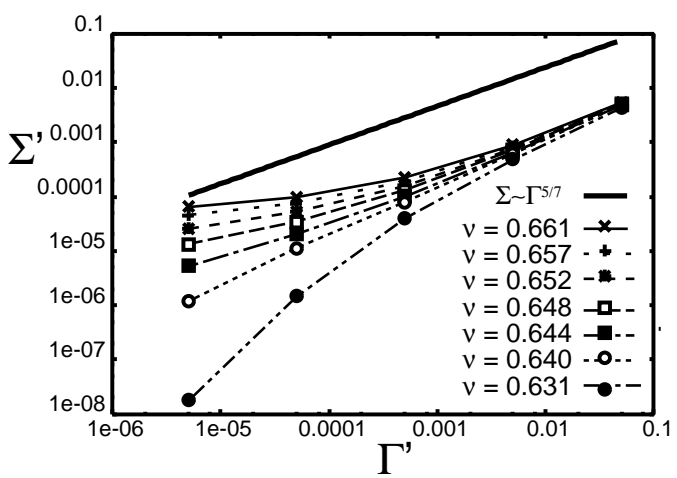

FIG. 6: Dimensionless shear stress $\Sigma^{\prime}$ as a function of the dimensionless shear rate $\Gamma^{\prime}$ for several values of $\nu$ with the interaction force $k^{\prime \prime}\left(\left(r_{1}+r_{2}\right)-\delta r\right)^{3 / 2}$. The dimensionless shear stress and the dimensionless shear rate are defined as $\Sigma^{\prime}=\sigma \sqrt{a} / k^{\prime \prime}$ and $\Gamma^{\prime}=\gamma \sqrt{m /\left(k^{\prime \prime} \sqrt{a}\right)}$, respectively. The parameter values are as follows: $N=1728$, and $\eta / \sqrt{k^{\prime \prime} m \sqrt{a}}=1.0$.

parameters. It might be important to develop a theory by using a more physical time scale such as the collision interval.

Finally, in our theoretical argument, Eq. (11) plays an essential role. As in the case of dense colloidal suspensions [15], one may investigate the pair-distribution function of granular systems in order to derive the order parameter equation. The establishment of a complete theory in which the scaling relations are derived from a microscopic model is an important topic for future studies.

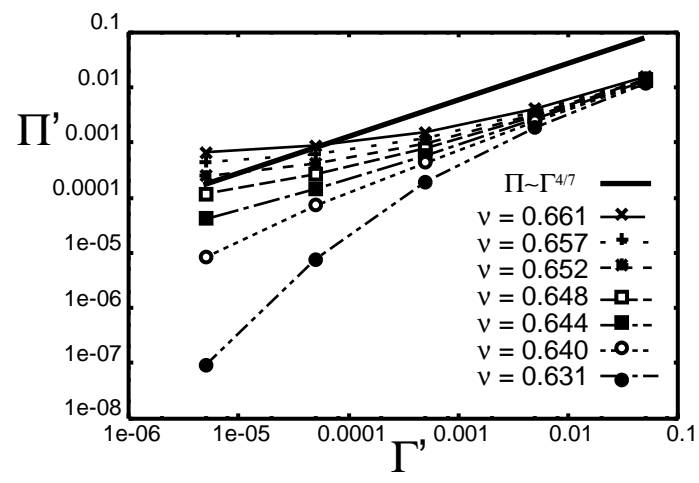

FIG. 7: Dimensionless shear stress $\Pi^{\prime}$ as a function of the dimensionless shear rate $\Gamma^{\prime}$ for several values of $\nu$ with the interaction force $k^{\prime \prime}\left(\left(r_{1}+r_{2}\right)-\delta r\right)^{3 / 2}$. The dimensionless shear stress and the dimensionless shear rate are defined as $\Pi^{\prime}=$ $p \sqrt{a} / k^{\prime \prime}$ and $\Gamma^{\prime}=\gamma \sqrt{m /\left(k^{\prime \prime} \sqrt{a}\right)}$, respectively. The parameter values are as follows: $N=1728$, and $\eta / \sqrt{k^{\prime \prime} m \sqrt{a}}=1.0$.

The authors thank H. Hayakawa, N. Mitarai, and S. Tatsumi for their useful comments on this work. This work was supported by a grant from the Ministry of Education, Science, Sports and Culture of Japan (No. 16540337).
[1] L. Berthier and J. -L. Barrat, J. Chem. Phys. 116, 6228 (2002).

[2] J. Rottler and M. O. Robbins, Phys. Rev. E 68, 011507 (2003).

[3] F. Varnik, L. Bocquet, and J. -L. Barrat, J. Chem. Phys. 120, 2788 (2004).

[4] C. S. O'Hern, L. E. Silbert, A. J. Liu, and S. R. Nagel, Phys. Rev. E 68, 011306 (2003).

[5] GDR Midi, Europhys. J. E 14, 341 (2004).

[6] N. Xu and C. S. O'Hern, Phys. Rev. E 73, 061303 (2006).

[7] T. Hatano, in preparation.

[8] Eq. (1) is obtained when a tangential force between interacting particles is ignored. For general cases where the tangential force is taken into account, the left-hand side of Eq. (1) is modified as $\sigma / p-\mu_{0}$, where $\mu_{0}$ corresponds to the maximum static friction constant. The value of the exponent $\phi$ is identical to that in the case of Eq. (1). The details will be reported elsewhere.

[9] D. J. Evans and G. Morris, Statistical mechanics of Nonequilibrium Liquids, (Academic, London, 1990).
[10] C. S. Campbell, J. Fluid Mech. 465, 261 (2002).

[11] A power-law behavior similar to that in Eq. (3) has been reported in the two-dimensional system [6], though the critical state is not focused on.

[12] A. Liu and S. Nagel, Nature (London) 396, 21 (1998).

[13] R. A. Bagnold, Proc. R. Soc. London A 225, 49 (1954).

[14] N. Mitarai and H. Nakanishi, Phys. Rev. Lett. 94, 128001 (2005).

[15] M. Otsuki and S. Sasa, e-print cond-mat/0511111

[16] We confirmed that a critical state was observed in the system with walls consisting of the same kind of particles as those in the bulk, by which the volume is maintained constant.

[17] C. Marone, Ann. Revs. Earth and Plan. Sci. 26, 643 (1998); F. Heslot, T. Baumberger, B. Perrin, B. Caroli, and C. Caroli, Phys. Rev. E 49, 4973 (1994).

[18] M. L. Blanpied, T. E. Tullis, and J. D. Weeks, Geophys. Res. Lett. 14, 554 (1987). 